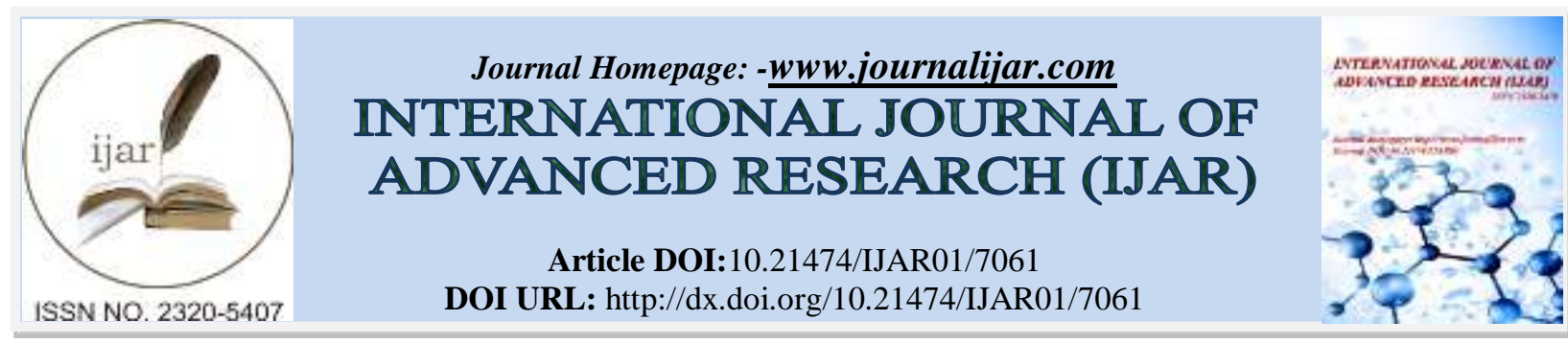

RESEARCH ARTICLE

\title{
IMPACT DES PRATIQUES DE GESTION DE LA FERTILITÉ DES SOLS SUR LA DIVERSITÉ, LE NOMBRE DE SPORES ET LES PARAMÈTRES DE MYCORHIZATION DU MIL.
}

\author{
Abdoul Razack Harouna Maidoukia ${ }^{1}$, Dahiratou Ibrahim Doka ${ }^{1}$, Abarchi Idrissa ${ }^{2}$, Gaston sangaré ${ }^{3}$ and \\ Moussa Barage. \\ 1 Laboratoire de mycologie Ecole Normale Supérieure Université Abdou Moumouni de Niamey Niger \\ 2 Faculté d'agronomie Université Dan Dicko Dan Koulodo de Maradi Niger \\ 3 International Crops Research Institute for the Semi-Arid Tropics (ICRISAT), Niamey, BP12404, Niamey, \\ Niger. \\ 4 Faculté d'agronomie Université Abdou Moumouni de Niamey Niger.
}

\section{Manuscript Info}

[.........................

Manuscript History

Received: 08 March 2018

Final Accepted: 10 April 2018

Published: May 2018

Keywords:-

endomycorhization,microbiologie

rhizosphère, pratiques culturales, sol, Niger.

\section{Abstract}

Les mycorhizes résultent d'une association durable basée sur des échanges réciproques entre les racines des plantes et certains champignons du sol. La présente étude a pour objectif d'évaluer l'effet des pratiques paysannes de gestion de la fertilité des sols sur les paramètres de mycorhization du mil ainsi que la densité et la diversité des spores de la rhizosphère. Pour cela des échantillons de sols et de racines ont été prélevés sur des parcelles mis en places. Les résultats de l'étude ont montré deux genres de spores de champignon à mycorhize arbusculaire, Glomus et Gigasporas avec une prédominance du genre Glomus. Pour ces deux genres de spores le nombre de Glomus $(42,7)$ et de Gigaspora $(14,11)$ est plus élevé au niveau du traiement paillage,et faible au niveau du parcage (Glomus 21,33 et 6,5 Gigasporas).la densité moyenne des spores est plus elevée au paillage $(56,83)$ qu'aux niveau des autres traitements fumier $(38,38)$, temoin $(33,61)$, parcage $(27,83)$.En ce qui concerne les paramètres de mycorhization la fréquence est de $100 \%$ dans tous les traitements. L'intensité de mycorhization ne montre pas de différence significative entre les traitements, cependant le témoin a enregistré le taux le plus important $(59,99)$, suivi du paillage $(59,51)$ et du fumier $(54,84)$, ce taux reste faible au niveau du parcage $(46,84)$. Pour la teneur en arbuscules, la valeur la plus élevée est observée au niveau du parcage $(14,92)$ et la plus faible au niveau du témoin $(7,3)$.

Copy Right, IJAR, 2018,. All rights reserved.

\section{Introduction:-}

L'agriculture nigérienne représente la principale activité de plus de $80 \%$ de la population active et se pratique sur une superficie n'excédant pas $12 \%$ du territoire (INS,2012). Elle reste largement tributaire des aléas climatiques et des chocs récurrents qui accroissent la vulnérabilité des populations aux crises alimentaires conjoncturelles assez fréquentes (I3N, 2012). De plus, la pauvreté des sols en matière organique aggrave la carence en phosphore car la minéralisation du phosphore organique joue un rôle vital dans la nutrition des plantes (Bationo et al, 1986 ; Amina, 
2007).Le sol est un environnement hétérogène complexe qui contient une grande variété de communautés et d'espèces bactériennes Daniel,(2005).Elles sont surtout abondantes autour des racines de certaines plantes (graminées, légumineuses) au sein de la rhizosphère Duchaufourd, (2001). Parmi ces microorganismes figurent les champignons mycorhiziens arbusculaires (CMA) qui vivent en symbiose avec les racines des plantes. Plusieurs auteurs ont montré l'importance de ces champignons symbiotiques dans l'améliorent de la nutrition minérale des plantes des sols pauvres (Leye et al., 2009 ; Bolan et al.,2011).Le rôle primordial des champignons à mycorhizes arbusculaires (CMA) se situe dans l'augmentation de l'absorption des éléments peu mobiles dans le sol, comme le Phosphore (P) et les métaux (Abbott et Robson, 1982). En effet, le champignon endomycorhizien constitue l'un des passages obligés entre les solutions minérales du sol et les plantes, et par la suite, contribue à une meilleure nutrition minérale. Beaucoup d'études ont montré que la plupart des plantes dépendent des mycorhizes pour une meilleure croissance dans le sol, à un niveau de fertilité donné (Jaizme-Vega et Azcon, 1995).Les bienfaits de l'association endomycorhizienne ne sont pas limités à la seule nutrition phosphatée. Généralement, l'endomycorhization augmente aussi l'absorption d'autres éléments minéraux comme le $\mathrm{K}, \mathrm{Ca}, \mathrm{Mg}$ et certains oligo-éléments tels que le Zn, S, Cu, etc (Oihabi et al., 1993 ; Meddich et al., 2000). Les associations mycorhiziennes peuvent jouer également un rôle significatif dans la décomposition et la minéralisation des matières organiques végétales et mobiliser les nutriments au bénéfice de laplante hôte (Gobat et al, 2003 ; Larnbers et al, 2008).He et Nara (2007) ont même suggéré que les mycorhizes pourraient jouer un rôle fondamental dans la réduction de la malnutrition humaine du fait que de nombreux nutriments se retrouvaient dans la biomasse des plantes mycorhizées (biofortification). Les champignons à mycorhizes arbusculaires CMA sont aussi des composantes essentielles pour le fonctionnement des écosystèmes naturels. Par la sécrétion de la glomaline, ils contribuent à l'amélioration et au maintien des agrégats de sols dans les zones érodées (Tisdall, 1991) par l'acquisition de meilleures propriétés physiques, chimiques et biologiques.Malgré le rôle important que jouent les mycorhizes dans l'amélioration de la structure du sol, peu d'intérêt leur est accordé au Niger; surtout quand ont sait que l'agriculture nigérienne est menacée par le phénomène de dégradation des terres se traduisant par une baisse de la fertilité des sols (INS, 2012).Cette étude réalisée en champs paysansdans la région périphérique du Parc W a pour but d'évaluer l'effet des pratiques paysannes de gestion de la fertilité des sols(parcage paillage apport du fumier organique et le témoin) sur les paramètres de mycorhization du mil, la densité et la diversité des spores de la rhizosphère.

\section{Matériel et methods:-}

\section{Site expérimentale:-}

L'étude a été menée en champs paysans dans la région périphérique du parc W (commune rurale de Tamou) situé à $75 \mathrm{~km}$ de Niamey, au cours des campagnes agricoles de 2015 et 2016, dans trois villages (Alambaré, Moli haoussa etLélédjé). Le dispositif expérimental est constitué de parcelles de $16 \mathrm{~m}^{2}$ disposées en blocs dispersés à raison d'un par champs. Les parcelles sont installées en fonction des traitements (parcage, paillage, apport du fumier organique et le témoin). Le matériel végétal utilisé est le mil (Pennisetum glaucum (L.)R.Br) variété locale etprincipale culture produite par les paysans de la zone du Parc W.

\section{Mesures et prélèvements:-}

Pour mener à bien les différentes observations et mesures au cours des essais, trois (3) poquets ont été choisis de façon aléatoire dans chaque parcelle utile. Les poquets choisis ont été ensuite matérialisés à l'aide de petits piquets. Le prélèvement des échantillons de sol et de radicelles au niveau de ces poquets ont été faits pendant les trois stades phénologiques du développement du mil (tallage, montaison, grenaison). Les prélèvements ont concerné uniquement les racines fines plus faciles à observer au microscope. Ces racines prélevées ont été conservées dans des boites numérotées contenant du GEE (Glycérol-Ethanol-Eau). Les échantillons de solont été prélevés au même endroit que les radicules et ont été mis dans des sachets plastiques stériles après séchage sous abri, à l'air libre puis transportés au laboratoire de mycologie de l'Ecole Normale Supérieure de l'université Abdou Moumouni de Niamey.

\section{Extraction et dénombrement des spores des champignons à mycorhizes arbusculaires:- Indigènes:-}

Les spores ont été extraites selon la méthode de Walker,(1982). Une quantité de 100g de sol a été versée dans un sceau rempli d'eau au 3/4. Après avoir agité pendant 10 à 15 seconds, la suspension est passée au travers de 5 tamis superposés dont les mailles respectives sont de $63 \mu \mathrm{m}, 160 \mu \mathrm{m}, 250 \mu \mathrm{m}, 315 \mu \mathrm{m}, 630 \mu \mathrm{m}$ en allant du bas vers le haut. Le dépôt dans le tamis de $630 \mu \mathrm{m}$ de maille est constitué de débris et contient rarement de spores, il a donc été jeté. Le refus des différents tamis restants a été recueilli dans des boites de pétri. Le contenu de ces dernières a été ensuite mélangé. Le comptage des spores à été fait sous une loupe binoculaire. 


\section{Estimation de l'infection des racines par les champignons à mycorhizes arbusculaires:-} Coloration des racines:-

La technique de coloration des racines utilisée est celle décrite par Philips et Hayman (1970) modifiée. Les racines sont lavées avec de l'eau puis conservées pendant $24 \mathrm{~h}$ dans une solution de potasse $(\mathrm{KOH})$ à $10 \%$. Elles sont ensuite, blanchies en ajoutant quelques gouttes de $\mathrm{H}_{2} \mathrm{O}_{2}(100 \mathrm{~V})$ au mélange $\mathrm{KOH}$ plus racines, pendant $5 \mathrm{mn}$. Après un rinçage à l'eau distillée, les racines sont acidifiées avec du $\mathrm{HCl}$ à $1 \%$ pendant quelques minutes. Elles sont à nouveau rincées à l'eau distillée, puis colorées au bleu trypan (pendant au moins $24 \mathrm{~h}$ à l'air ambiant). Les racines sont enfin rincées à l'eau distillée et conservées dans une petite quantité de glycérine.

\section{Evaluation des paramètres de mycorhization:-}

Pour chaque échantillon, 30 fragments d'un (1) cm de long des racines colorées sont découpés et montés entre lame et lamelle en raison de 10 fragments par lame. Les lames sont observées au microscope ; chaque fragment est soigneusement examiné sur toute sa longueur pour la détection des paramètres de mycorhizations ; la méthode d'évaluation utilisée est celle proposée par Trouvelot et al., (1986). Ces paramètres sont :

Fréquence de mycorhization( F\%):-

La fréquence de mycorhization est définie comme le pourcentage de fragments racinaires mycorhizés par rapport au nombre total de fragments observés

\section{L'intensité de mycorhization (M\%):-}

L'intensité de mycorhization correspondant à la proportion de fragments racinaires observés colonisés par le CMA.

\section{La teneur en arbuscule (A\%):-}

La teneur en arbuscules correspondant à la proportion de fragments racinaires colonisés par les arbuscules.

\section{Traitement statistique:-}

Les données collectées des différents paramètres ont été soumises à une analyse de variance (ANOVA) à l'aide du logiciel Minitab, version 16 et les moyennes des variables ont été comparées en utilisant le test de Tukey au seuil de probabilité $\mathrm{p}=5 \%$.Le logiciel Mycocalc, a servi pour l'évaluation des paramètres de mycorhization ( $\mathrm{F} \%$, M\% et A\%).

\section{Résultats:-}

I. Dénombrement et identification des spores des champignons à mycorhizes arbusculaire:-

Le tableau1 montre les caractéristiques des spores dans les différents traitements. La majorité des spores présentent

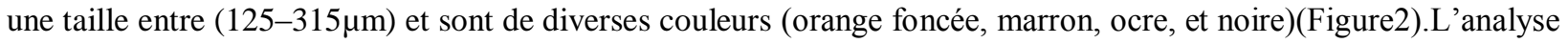
de ces spores fait ressortir deux genres, Glomus (Figure2 a) et Gigasporas (Figure2 b, c)avec une prédominance du genre Glomus dans tous les traitements. Ces spores appartiennent respectivement à la famille des Glomaceae et Gigasporaceae.

\section{Impact des traitements sur le nombre et le genre de spores:-}

La figure 3 décrit les effet des traitements sur le nombre de spores en fonction des genres.

L'analyse de la variance $(\mathrm{p}=5 \%)$ a révélé des différences significatives entre les traitements pour les deux genres de spore. Le nombre de Glomus $(42,7)$ et de Gigaspora $(14,11)$ est plus élevé au niveau du traiement paillage. Par contre aucune difference significative n'a été constatée entre les traitements comportant le fumier (Glomus 27,77 et 10,61 Gigasporas) et le temoin (Glomus 24,55 et 9,05 Gigasporas). les plus faibles valeurs ont été obtenues au niveau du traitement parcage (Glomus 21,33 et 6,5 Gigasporas).

Impact des traitements sur la densité des spores de la rhizosphère des plants de mil:-

La densité moyenne des spores enregistrées dans $100 \mathrm{~g}$ de sol est représentée par la Figure 4. L'analyse de la variance montre une difference hautement significative entre la densité moyenne des spores du traitement paillage $(56,83)$ et la densité des spores des traitements fumier $(38,38)$, temoin $(33,61)$, parcage $(27,83)$.La taille des boites à moustaches et les écarts interquartiles(Figure 4) traduisent la variation et la distribution des spores de CMA autour de la rhizosphère du mil dans chaque traitement ; ainsi les spores du parcage $(E$. Int 15,25$)$ et du paillage (E. Int 13,76) sont équitablement reparties dans la rhizosphère. Cependant malgré des densités moyennes élevées de spores au niveau du fumier et du témoin, il ya une faible répartition de celles-ci dans la rhizosphère. $(E$. Int 9,25$)$ et $(E$. Int 
7). Il a été observé une différence du nombre de spore en fonction des années ainsi la figure 5montre une densité plus élevé de spores en 2016 par rapport à 2015.

\section{Paramètres de mycorhization:-}

L'examen des racines du mil a montré que tous les échantillons observés au microscope sont colonisés par des champignons mycorhiziens arbusculaires (CMA). La fréquence de mycorhization (F\%) a atteint les $100 \%$ au niveau des racines de mil de tous les traitements. Pour l'intensité de mycorhization (M\%), l'analyse de la variance (Figure 6)ne montre pas de différence significative entre les traitements; cependant on constate que le témoin a enregistré le taux le plus important $(59,99)$, suivi du paillage $(59,51)$ et du fumier $(54,84)$. Ce taux reste faible au niveau du parcage $(46,84)$.En ce qui concerne la teneur en arbuscules (A\%), la valeur la plus élevée est observée au niveau du parcage $(14,92)$ et la plus faible au niveau du témoin $(7,3)$.

\section{Discussion:-}

L'analyse des échantillons de sol a permis d'identifier deux genres de spores dont une appartient à la famille des Glomus et l'autre à la famille des Gigasporacées. Laminou, (2010) et Ibrahim et al., (1995) ont déjà trouvé ces deux genres de spores dans des études antérieure menées au Niger. Les résultats montrent également une prédominance du genre glomus confirmant ainsi ceux d'Abdoulaye,(2013) à Sadoré qui stipule que le genre Glomus est plus représentatif que le genre Gigaspora.Meddich et al, (2017) dans leurs études sur l'évaluation des potentiels mycorhizogens du sol ont trouvés que le genre Glomus est plus rependu que les autres genres de spores. La densité moyenne des spores contenues dans les sols par traitement varie de 27 à 56. Ces nombres sont nettement inférieurs à ceux obtenus par Laminou, (2010) dans la zone de Gouré. Selon cet auteur, la moyenne des spores dans 100g de sol est de 156 pour le mil et 107 pour le sorgho. Le faible nombre de spore récolté dans la rhizosphère des plants pourrait être dû au travail du sol, le labour est pratiqué par la majorité des agriculteurs des villages de la zone du Parc W. En effet, le labour provoque un enfouissement des organes mycorhiziens et ralentit la colonisation et l'exploration du sol (Goss et de Varennes, 2002; Kabir, 2005).Les résultats de l'analyse des racines du mil prélevées ont montré une fréquence de mycorhization proche des $100 \%$ au niveau des racines de tous les traitements. L'intensité de mycorhization montre une légère différence entre les traitements, mais plus élevée au niveau du témoin et du paillage. Cette intensité de mycorhization élevée au niveau du témoin et du paillage pourrait s'expliquer probablement par la faible concentration en éléments minéraux dans ces deux traitements; ce qui favoriserait l'infection racinaire des CMA. En effet, le bon fonctionnement de la mycorhization est relevé dans le cas des sols pauvres surtout en phosphore. C'est sous des conditions déficitaires que la plante hôte établit cette relation symbiotique avec les CMA et facilite le plus les échanges avec le partenaire mycorhize Meddich et al, (2017).Le taux le plus faible est observé au niveau du parcage. L'intensité de la colonisation racinaire par les champignons symbiotiques est réduite quand le niveau de phosphore augmente dans le sol et devient ainsi directement disponible pour la plante Dickson et al., (1999).Selon Garcia et al., (2001) une concentration élevée en phosphore et en azote conduirait à une diminution de la disponibilité en sucres solubles au niveau de la racine, d'où une réduction de l'intensité de mycorhization. Cependant pour la teneur en arbuscules il a été constaté que la valeur la plus élevée est enregistrée au niveau du parcage. Cela pourrait s'expliquer par le fait que les traitements comme le parcage nourris le sol avec beaucoup de bactéries et non la plante. Selon Grosling et al, (2006) La fumure organique n'est pas néfaste au développement des mycorhizes et peut même les stimuler.

\section{Conclusion:-}

Ce travail a permis de voir l'impact des pratiques de gestion de la fertilité du sol sur les paramètres de mycorhization des racines du mil ainsi que la densité des spores de la rhizosphère. L'analyse des résultats sur l'identification et le dénombrement des spores ont permis d'identifier deux types de spores (Glomus et Gigaspora) avec la prédominance du genre Glomus. Les résultats obtenus montrent une faible densité de spore dans les différents traitements. Cependant cette densité varie selon les traitements, ces spores ont étés beaucoup plus abondantes au niveau du paillage que dans les autres traitements. Pour ce qui est des paramètres de mycorhization, l'étude a permis de mettre en évidence l'effet positif des traitements sur la fréquence de mycorhization qui est de $100 \%$ dans tous les traitements. L'étude a permis également de montrer que l'intensité de mycorhization et la teneur arbusculaire sont fonction des pratiques de gestion de la fertilité des sols. 


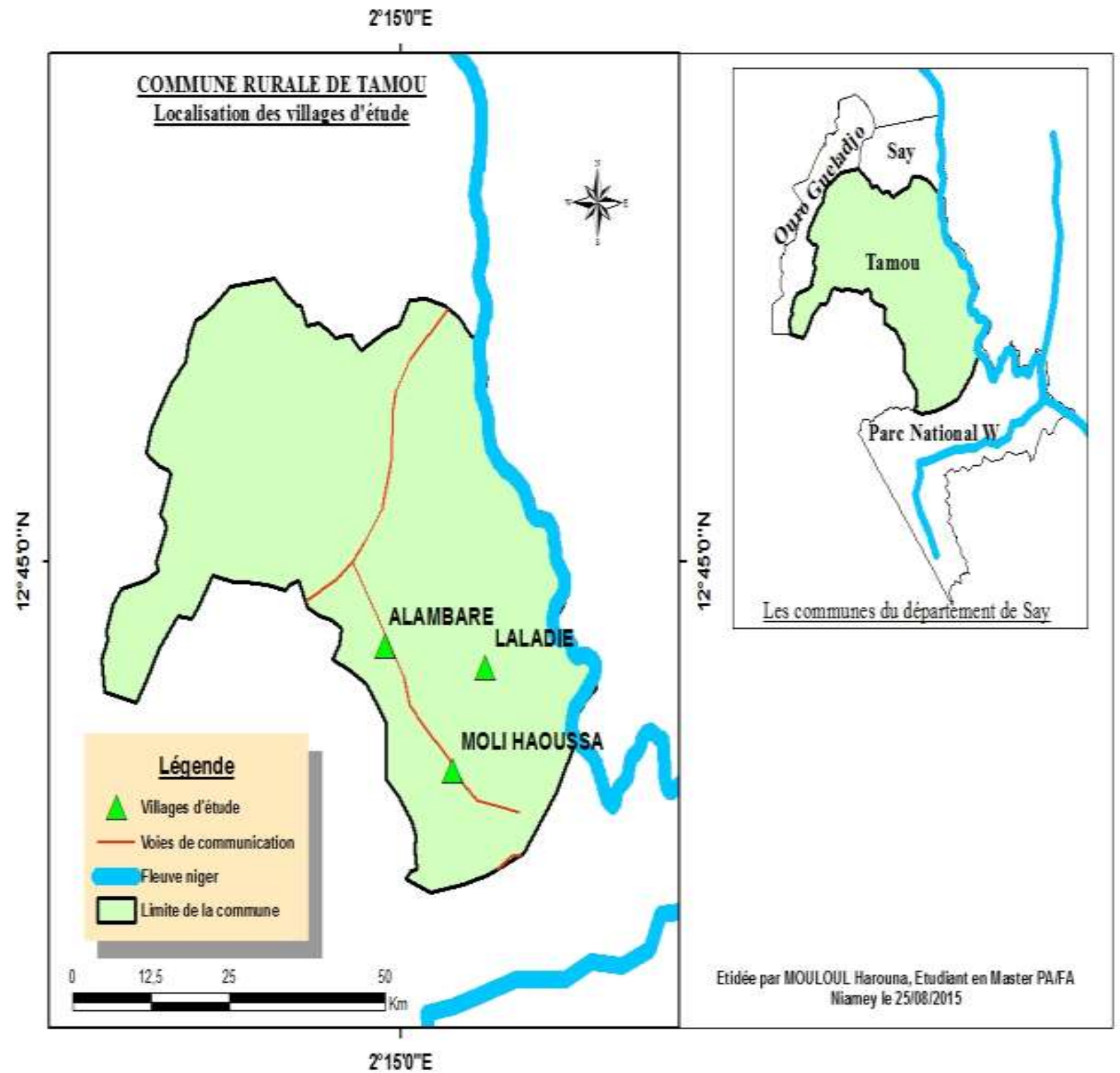

Figure1:-cartographie de la commune rurale de Tamou (les triangles verts sur la carte designent les villages abritant les sites de l'etude)

Tableau 1:-caracteristiques des spores par traitement

\begin{tabular}{|l|l|l|}
\hline Traitements & Couleur des spores & Taille des spores \\
\hline Parcage & $\begin{array}{l}\text { Orange foncée, } \\
\text { marron, et noire }\end{array}$ & $\begin{array}{l}\text { Structures sphériques } \\
(125-315 \mu \mathrm{m})\end{array}$ \\
\hline Paillage & Ocre, noire, orange & Structures sphériques $(125-315 \mu \mathrm{m})$ \\
\hline Fumier & Noire, marron & $\begin{array}{l}\text { Structures sphériques, ovales } \\
(125-315 \mu \mathrm{m})\end{array}$ \\
\hline Témoin & $\begin{array}{l}\text { Jaune, orange, } \\
(125-315 \mu \mathrm{m})\end{array}$ \\
\hline
\end{tabular}




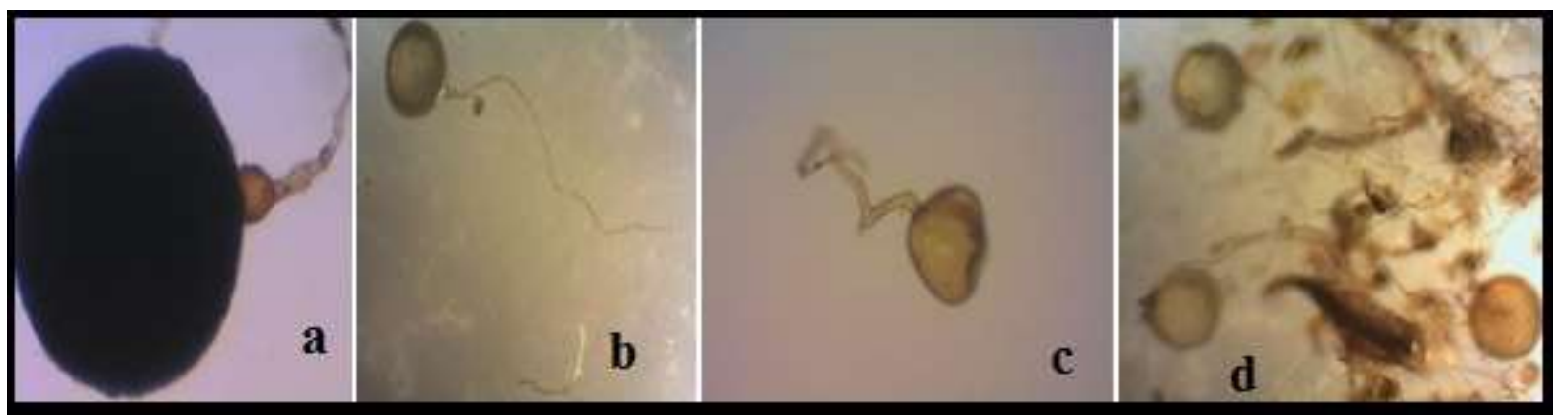

Figure2:-Genres de spores isolées à partir des traitements

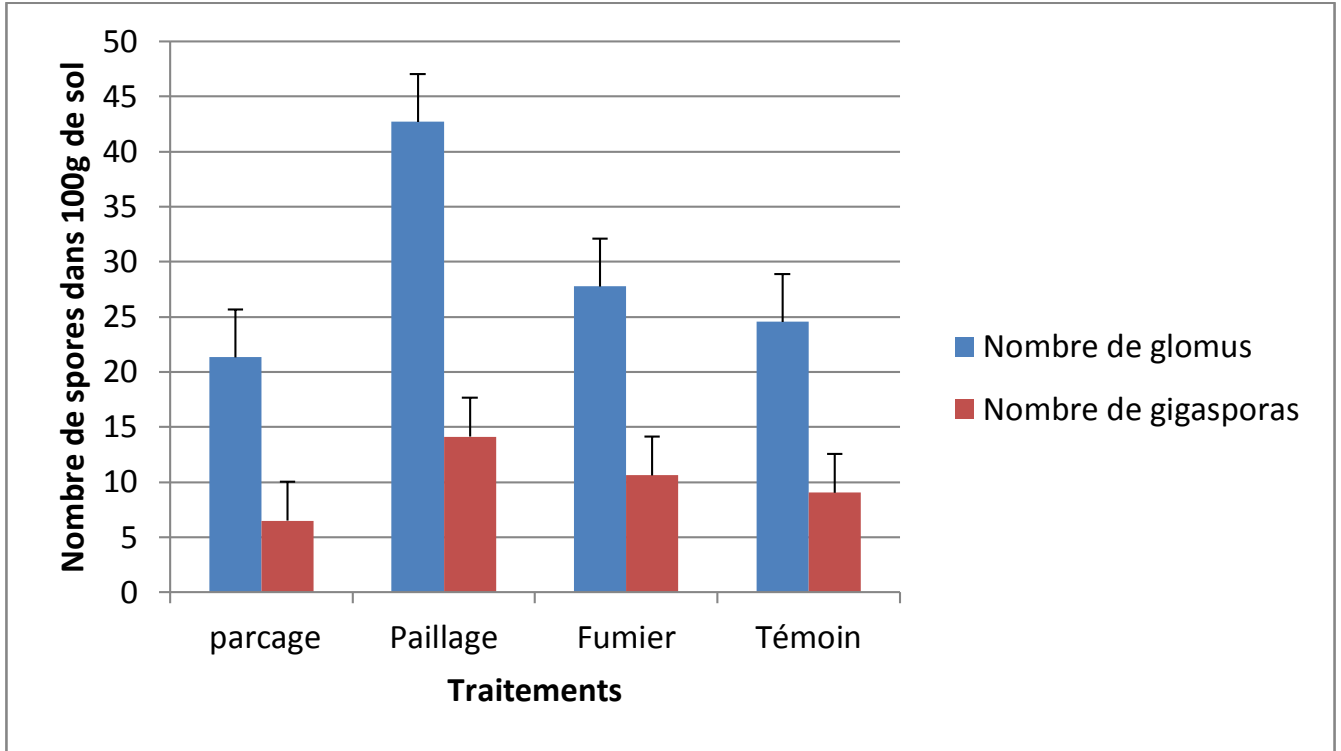

Figure 3:-Nombre de spores par traitement 


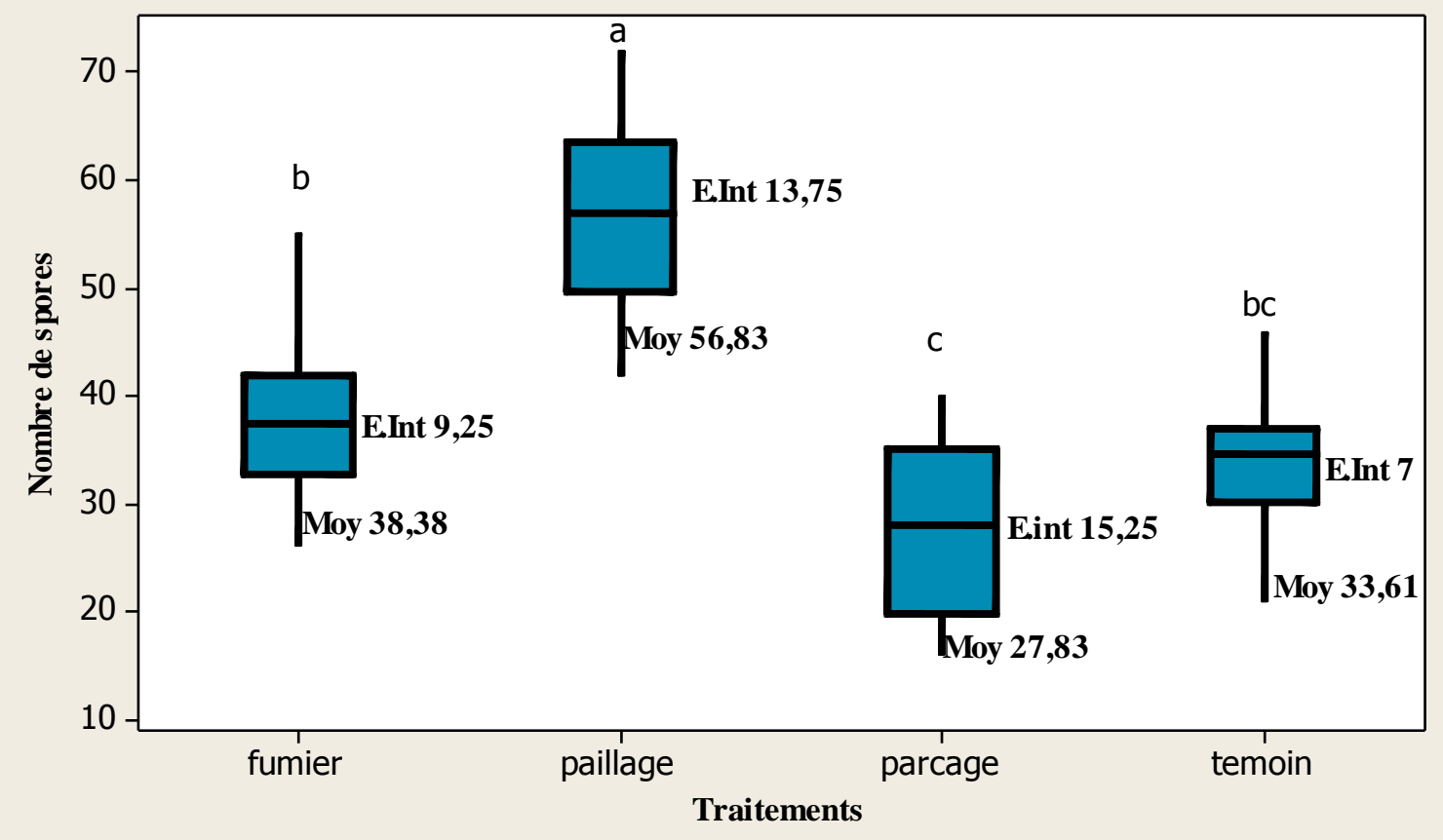

Figure4:-Densité et variation du nombre des spores par traitements : Les moyennes ne partageant aucune lettre sont sensiblement différentes au seuil de $5 \%$.

E.int (Ecart interquartiles), Moy (moyenne)

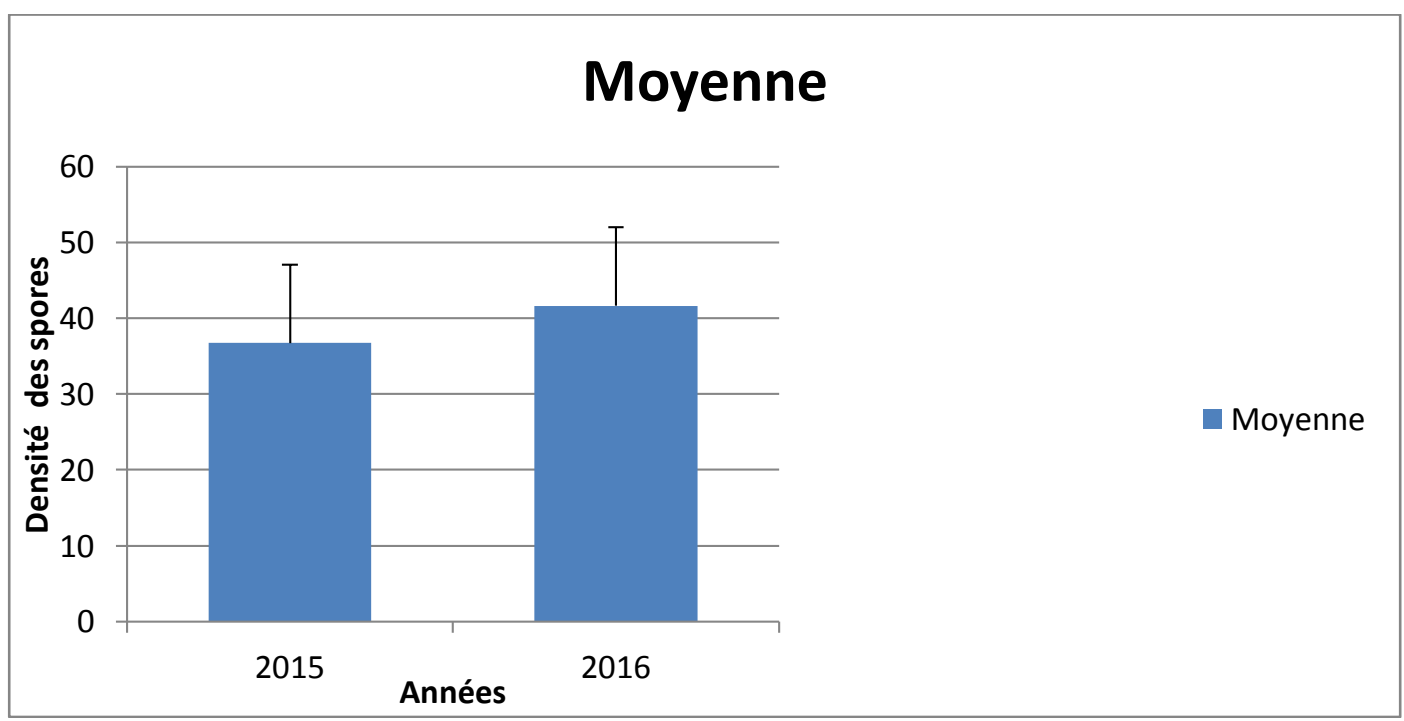

Figure 5:-Densité des spores par années et par années 


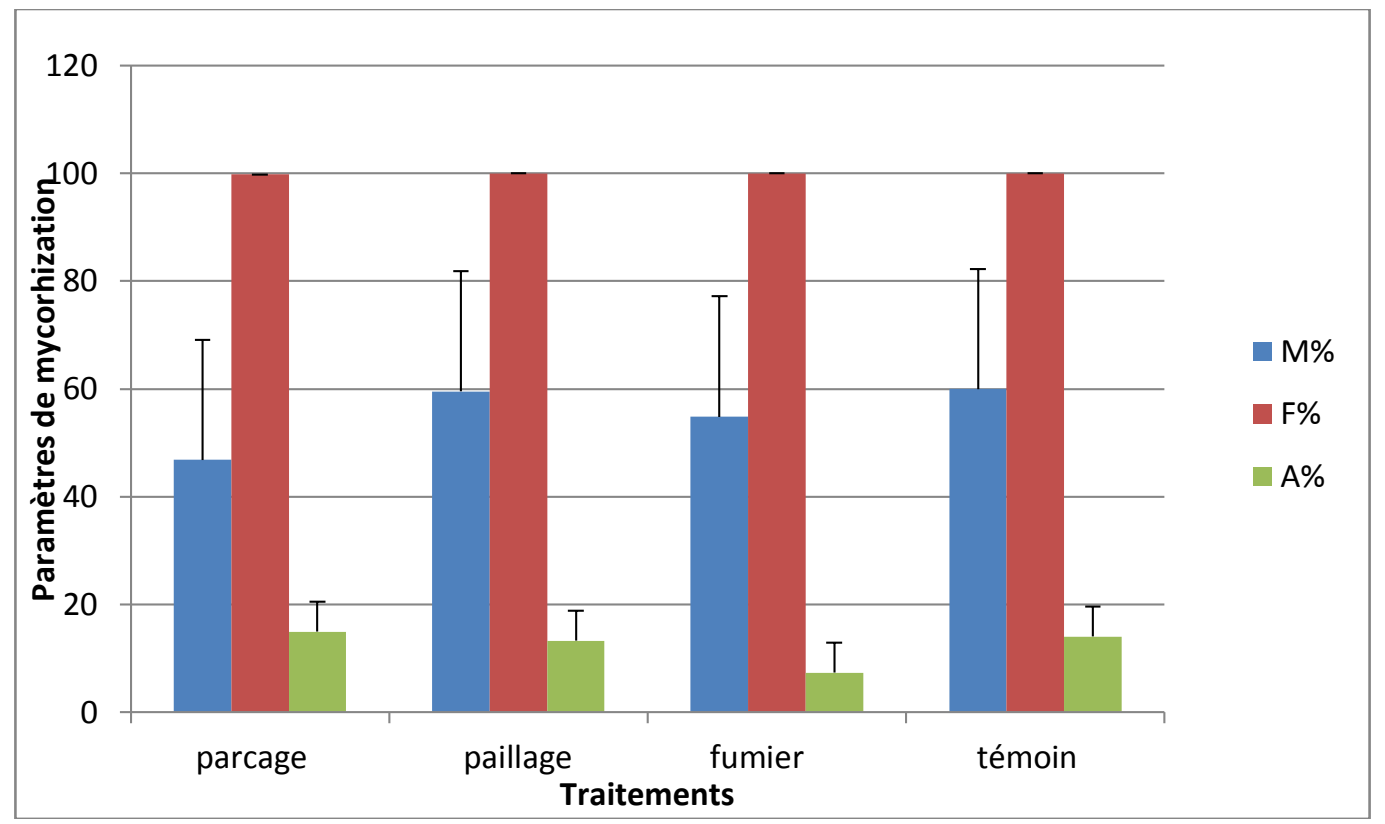

Figure 6:-Intensité, fréquence et taux arbusculaire par traitements

\section{Reference:-}

1. Amina G.,2007 : Production d'inoculum des champignons à mycorhizes arbusculaires en conditions paysannes nigériennes ; Université Abdou Moumouni de Niamey/ FA.32 P

2. Abdoulaye A.,2013 : Etude comparative de l'influence de la densité de semis sur la mycorhization du Voandzou (Vigna subterranea L.), de la Dolique (Dolichos lablab L.) et du Mil (Pennissetum glaucum L.) dans la gestion de la fertilité des sols ; Université Abdou Moumouni de Niamey/ FA. 56 P

3. Abbott, LK et Robson, AD 1982. Le rôle des champignons mycorhize VAl'agriculture et la sélection des champignons pour l'inoculation. Aust.J. Agric.

4. Res.33: 389

5. Bolan, N. S., Adriano, D., Senesi, N., Kunhikrishnan, A., James, T., and McDowell,

6. R.(2011). Dissolved organic carbon: Biogeochemistry, dynamics and agro-environmental significance in soils.Adv. Agron.110, 1-75.

7. Daniel R. 2005. The metagenomics of soil.

8. Nature Reviews Microbiology3: 470-8 Das S, Jean J-S, Kar S, Liu C-C. 2012. Changes in Bacterial Community Structure and Abundance in Agricultural Soils under Varying Levels of Arsenic Contamination. Geomicrobiology Journal30: 635-44

9. Dickson, D. W., Lin, W., Liu, W. K., and Yen, S. H. (1999). Multiple system atrophy: a sporadic synucleinopathy. Brain Pathol. 9, 721-732. doi: 10.1111/j.1750-3639.1999.tb00553.

10. Duchaufourd PH., 2001. Introduction à ma science du sol. 6èmeEdit de l'abrégé de pédologie.Dunod. Ed. Massson. Paris. P314.

11. Graham J.H.and Syvertsen J.P., 1989. Vesicular-arbuscular mycorrhizas increase chloride concentration in citrus seedlings.NewPhytol., $113: 29-36$.

12. Gosling, P., Hodge, A. Goodlass, G. and Bending, G. D. (2006). Review: Arbuscular mycorrhizal fungi and organic farming. Agriculture,Ecosystems and Environment, vol. 113, p. 17 -35.

13. Gobat,J.M.,Aragno,M.,Matthey,W.(2003). Le sol vivant, $2^{\mathrm{e}}$ Edition. Presses Polytechniques Universitaires Romandes, Lausanne, 58p

14. Goss, M.J. \& de Varennes, A. 2002. Soil disturbance reduces the efficacy of mycorrhizal associations for early soybean growth and N2 fixation. Soil Biology and Biochemistry, 34, 1167-1173.

15. Garcia J-L., Roger PA (2001), Introduction à la microbiologie du sol. Polycopié de cours (Université de Provence, Université de la Méditerranée, Ecole Supérieure d'Ingénieurs de Luminy. 191pp

16. Harley J.L.and Smith S.E., 1983. Mycorrhizal Symbiosis. Academic Press, Londres, $483 \mathrm{p}$

17. He,X.,Nara,K.(2007).Element biofortification: can mycorrhizas potentially offer a more effective and sustainable pathway to curb Human malnutrition? Trends in Plant Science,12:331-33 
18. Initiative 3N, 2012. Pour la sécurité alimentaire et nutritionnelle et le développement agricole durable. Hautcommissariat à l'initiative $3 \mathrm{~N}$. Niger, 69 pages.

19. Ibrahim D, Pierart P, Theon D. 1995. Premières observations sur les mycorhizes vésiculo-arbusculaires de la rhizosphère de quelques spermaphytes du Sud-Ouest du Niger. Belgi. J. Bot., 28: 151-164.

20. Jaizme-Vega M.C. \& R. Azcon. 1995. Responses of some tropical and subtropical cultures to endomycorrhizal fungi. Mycorrhiza 5: 213217.

21. Kabir, Z. 2005. Tillage or no-tillage: impact on mycorrhizae. Canadian Journal of Plant Science, 85, $23-29$.

22. Lambers,H.,Raven,J.A.,Shaver,G.R,Smith,S.E.(2008).Plant nutrient-acqnisition strategies change with soil age. Trends in Ecology, Evolution,23:95-103.

23. Laminou MO. 2010. Fixation des dunes dans le Sud-Est du Niger: évaluation de l'efficacité de la barrière mécanique, espèces ligneuses adaptées et potentialités d'inoculation mycorhizienne. Thèse de doctorat en Sciences agronomiques et ingénierie biologique, Université de Liège, p. 142.

24. Leye EHM, Ndiaye F, Diallo B, Sarr AS, Diouf M, Diop T. 2009. Effet de la mycorhization sur la croissance et le développement de Jatropha curcas L. Rev Energ. Renouvel., 12(2): 269-278.

25. Meddich A., Oihabi A., Abbas Y.and Bizid E. 2000. Rôles des champignons mycorhiziens à arbuscules des zones arides dans la résistance du trèfle (Trifoliumalexandrinum) au déficit hydrique. Agronomie 20 : 283-295.

26. Meddich A, Ait El Mokhtar M,Wahbi S, Boumezzough A. 2017. Évaluation des potentialités mycorhizogènes en lien avec les paramètres physico-chimiques des sols de palmeraies du Maroc (Marrakech et Tafilalet). Cah. Agric. 26: 45012.

27. Oihabi A., Perrin R.and Marty F., 1993. Effets des endomycorhizes VA sur la croissance et la nutrition du palmier dattier. Rev.Rés.Amelio. Prod. Agr. Milieu aride, 5: pp. 1-9.

28. Philips JM, Hayman DS (1970). Improved procedure for clearing roots and staining parasitic and vesiculararbuscular mycorrizal fungi for rapid assessment of infection. Trans. Br. Mycol. Soc. 55:158-161. Power JF, Koerner P, Doran JW, Wilhelm W (1998). "Residual Effects of Crop Residues on Grain Production and Selected Soil Properties". Publications from USDA-ARS / UNL Faculty. Paper 89. http://digitalcommons.unl.edu/usdaarsfacpub/89

29. Tisdall J.M., 1991. Fungal hyphae and structural stability of soil. Aust. J. Soil Res., 29: 729-743.

30. Trouvelot A, Kough JL, Gianinazzi V (1986). Mesure de taux de mycorrhization VA d'un système radiculaire. Recherche de méthodes d'estimation ayant une signification fonctionnelle. In physiological and genetic aspects of mycorhizical, V. Gianinazzi-Pearson et S. Gianinazzi). (édit), INRA, Paris. pp. 217-221.

31. Walker C (1982). Systematics and taxonomy of arbuscular endomycorhizal fungi (Glomales) a possible way forward. Elsevier/INRA. Agronomie 12:887-897. Warner A, Mosse B (1982). Factors affecting the spread of vesicular mycorrhizal fungi in soil. New Phytologist 90:529-536. 